\title{
Synthesis and Properties of Anion Exchangers Derived from Chloromethyl Styrene Codivinylbenzene and Their Use in Water Treatment
}

\author{
Hesham A. Ezzeldin, ${ }^{1}$ Allen Apblett, ${ }^{2}$ and Gary L. Foutch ${ }^{3}$ \\ ${ }^{1}$ Hydrogeochemistry Department, Desert Research Center, Mataria, Cairo 11753, Egypt \\ ${ }^{2}$ Department of Chemistry, Oklahoma State University, Stillwater, OK 74078, USA \\ ${ }^{3}$ School of Chemical Engineering, Oklahoma State University, Stillwater, OK 74078, USA \\ Correspondence should be addressed to Gary L. Foutch, foutch@okstate.edu \\ Received 8 January 2010; Accepted 5 May 2010 \\ Academic Editor: Marek Cypryk
}

Copyright ( 2010 Hesham A. Ezzeldin et al. This is an open access article distributed under the Creative Commons Attribution License, which permits unrestricted use, distribution, and reproduction in any medium, provided the original work is properly cited.

\begin{abstract}
Amination of vinylbenzyl chloride-divinylbenzene (VBC-DVB) copolymers is an effective method for preparation of ionexchange resins. Conventionally, the starting polymer is produced by chloromethylation of a styrene-divinylbenzene copolymer that utilizes chloromethyl methyl ether, a known carcinogen. An alterative approach is to copolymerize vinylbenzyl chloride with divinylbenzene to generate the necessary VBC-DVB. This method provides precise control over the density of the ionexchange groups. The regiochemistry of the vinylbenzyl chloride methods was realized using solvent-ion exchange groups. In this investigation, an improved solvent system was found for the preparation of anion exchange resins by the vinylbenzyl chloride route. The effectiveness of amination of the intermediate VBC-DVB polymers with a variety of trimethylamine reagents was investigated, and ethanolic trimethylamine produced the highest degree of amination. These resulting ion-exchange polymers were characterized by a variety of techniques such as analytical titrations, nitrogen analysis, Fourier transform infrared spectroscopy and thermal gravimetric analysis. Testing of these copolymers for breakthrough was performed. The results indicate that these anion exchangers have a meaningful increase in thermal stability over commercial anionic exchange beads.
\end{abstract}

\section{Introduction}

Ion exchange membranes (IEMs) can be used for numerous applications including desalination of seawater, softening of hard water, recovery of metal ions, and purification of products and water $[1,2]$. Compared with cation exchangers, the anion exchange membranes have less discussion in the literature despite their growing importance for application in retention of multivalent ions and recovery of valuable metals from the effluent of metal plating industry [3]. Conventionally, anion exchangers based on cross-linked polystyrene are prepared by a method that involves chloromethylation of a styrene/divinylbenzene copolymer using either chloromethyl methyl ether or bis chloromethyl ether as the alkylating reagent. Subsequent treatment with amines then introduces the ion-exchange functionality into the polymer [4]. However, both of the alkylating agents have been reported to be strongly carcinogenic agents [5], and their use was seriously restrained in 1967 [6]. Additionally, the chloromethylation reaction also leads to further cross-linking that is detrimental to the performance of the resins [7]. This secondary crosslinking occurs through methylene group bridging and is thought to affect the capacity of the ion-exchange resin and increase its fragility [8].

The use of chloromethylating reagents can be avoided by synthesizing the desired vinylbenzyl chloride/divinylbenzene copolymer utilizing vinylbenzyl chloride as a starting material [9]. This approach was used in this investigation in which the influence of the polymerization solvent and the divinyl benzene content on the ion-exchange end product was determined. The objective was to prepare anion exchangers with improved characteristics over commercial anion exchange beads that are synthesized via a suspension polymerization of a styrene-divinylbenzene matrix. 
In the subsequent amination step, the chloromethylated copolymer is conventionally swelled in a suitable solvent such as dioxane, butanone, tetrahydrofuran, or benzene to allow for a uniform reaction and to help reduce the effects of osmotic shock $[4,10]$. In this investigation, amination without a solvent and with more environmentally friendly solvents such as water and ethanol was evaluated. Notably, the amination reaction time can be reduced to 4 days, as compared to 7 days for the commercial beads (the quaternization reaction of the commercial beads with $7.5 \%$ divinylbenzene concentration required 7 days at $0{ }^{\circ} \mathrm{C}$ using anhydrous amines [4]).

In addition to the synthesis and characterization of the ion exchange systems, an area of specific interest for this study was the removal of excess nitrate, phosphate, sulfate, and chloride ions from brackish and moderately saline waters. These anions, along with cations such as sodium, calcium, and magnesium, contribute to the salinity of water. It is worth mentioning that little work has been done using anion exchange resins for multianion removal from water. Among these are the removal of arsenic $[9,11,12]$ and other anions using different commercial organic resins [13].

\section{Experimental}

2.1. Materials. The starting monomer used in this work was vinylbenzyl chloride (VBC) $90 \%$ (Aldrich) that is a mixture of para and meta isomers. Divinylbenzene (DVB), $80 \%$ (Sigma-Aldrich), was used as a cross-linker, and benzoyl peroxide (Luperox A98) reagent grade, $\geq 98 \%$ (SigmaAldrich), was used as a radical initiator. The following chemicals or solutions were used as functionalizing agents: trimethylamine solution purum, 31-35 wt.\% in ethanol ( $4.2 \mathrm{M})$ (Sigma-Aldrich), trimethylamine solution purum, $\sim 45$ wt. $\%$ in $\mathrm{H}_{2} \mathrm{O}$ (Sigma-Aldrich), pure trimethylamine (Sigma-Aldrich), and triethylamine (Sigma-Aldrich). All of the aforementioned chemicals were used without further purification. Other solvents were reagent grade and used as received.

2.2. Ion Exchanger Synthesis. A mixture of vinylbenzyl chloride (VBC) and divinylbenzene in a ratio of 6.2 to 1 by weight was dissolved in a solvent mixture, and then $3 \%$ benzoyl peroxide was added as a radical initiator. Tetrahydronaphthalene (THN), Xylene, and a $1: 1 \mathrm{v}: \mathrm{v}$ mixture of tetrahydronaphthalene and xylene were used as solvents in order to determine the influence of these on the resulting polymer. In all cases, the amounts were $62 \% \mathrm{VBC}, 10 \%$ DVB, 3\% initiator, and 25\% solvent by weight. Radical polymerization was carried out by heating the solutions for $10 \mathrm{~h}$ at $80^{\circ} \mathrm{C}$ to convert them to gels. The resulting copolymers were ground and freed from the polymerization by-products, any homopolymer that may have formed, and the polymerization solvent by extraction with methanol in a Soxhlet apparatus for $24 \mathrm{~h}$. They were then dried in an oven at $40^{\circ} \mathrm{C}$ for $24 \mathrm{hr}$ and weighed. The reaction is presented diagrammatically in Figure 1.

To determine the influence of the degree of cross-linking (Figure 2), polymers were prepared using $2.5 \mathrm{~g}$ of both THN and xylene as solvent with the amount of divinylbenzene being adjusted to $6,8,10$, and $12 \%$ by weight following the same procedure described above (total mixture weight was $10 \mathrm{~g}$ ). The yields of the four polymers were 6.4, 6.1, 6.1, and $5.7 \mathrm{~g}$, respectively.

The copolymers were functionalized by reaction with trimethylamine gas and trimethylamine solutions in ethanol and water at $60^{\circ} \mathrm{C}$ for four days. An excess of trimethylamine was used in comparison to the chloromethyl content of the copolymer. Typically, $3 \mathrm{~g}$ of polymer was reacted with $30 \mathrm{~mL}$ of solution. In the case of the gas-phase reaction, a volume of $250 \mathrm{~mL}$ of trimethylamine was used. The resulting resins were washed with water/methanol, and water and dried at $55^{\circ} \mathrm{C}$ in a vacuum oven until they reached a constant weight (typically 12 hours). The yields were $3.035,3.32$, and $3.34 \mathrm{~g}$ for the solventless, aqueous, and ethanolic reactions, respectively. A reaction was also performed between the copolymer and neat triethylamine using the same procedure.

\subsection{Characterization}

2.3.1. Swelling Measurements. Uptake of water was determined by the difference in weight between the water-swelled and the dried polymer. Swelling is represented as milligrams of water per gram of dry resin. The water uptake (WU) was calculated by the following equation:

$$
\mathrm{WU}=\frac{\left[W_{\text {wet }}-W_{\text {dry }}\right] \times 100}{W_{\text {dry }}} .
$$

2.3.2. Thermogravimetric Analysis (TGA). Copolymers were dried at $70^{\circ} \mathrm{C}$ for $5 \mathrm{~h}$ before analyzing them. TGA runs were performed with a Seiko TGA/DTA Instrument model 6200 in the range of $25-400^{\circ} \mathrm{C}$ under air atmosphere. A mass of

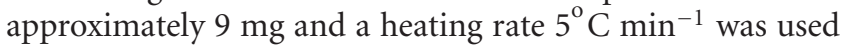
for all the samples.

2.3.3. Degree of Amination (\%). The aminated polymers were characterized by combustion analysis to determine the nitrogen content. The degree of amination and the amine group density were subsequently calculated. The upper limit of the expected nitrogen content should be $6.26,6.18,6.08$, and $5.96 \%$ for the $6,8,10$, and $12 \%$ DVB resins, respectively, assuming the functional groups in VBC completely reacted with trimethylamine.

2.3.4. Ion-Exchange Capacity. A strong base anion-exchange resin generally has a greater affinity for more highly charged anions over lower-charged ones [9]. Immersion of the membrane in an $\mathrm{NaOH}$ solution causes hydroxide ions to replace the chloride ions that are associated with the trimethylammonium groups of the ion exchange resin. Therefore, the ion-exchange capacity of the resins was determined by immersing in $0.1 \mathrm{~mol} \mathrm{~L}^{-1} \mathrm{NaOH}$ solution for 24 h. The hydroxide depleted from the solution during the ionexchange process was determined by titration against $0.1 \mathrm{~mol}$ $1^{-1} \mathrm{HCl}$ solution using a Titronic electronic burette and phenolphthalein as indicator. After titration, the polymers 
<smiles>C=Cc1cccc(Cl)c1</smiles>

Vinylbenzyl chloride (VBC)<smiles>C=Cc1ccc(C=C)cc1</smiles>

Divinylbenzene (DVB)

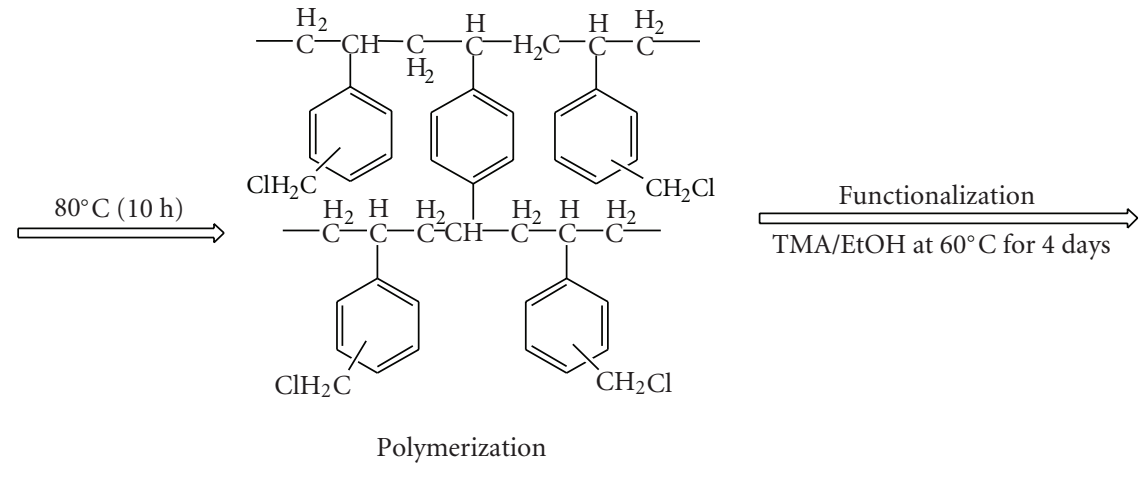

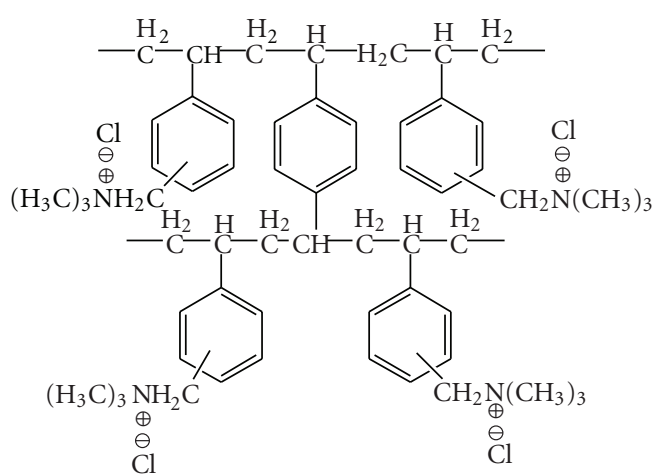

FIGURE 1: Polymerization and functionalization of anion exchange polymer.

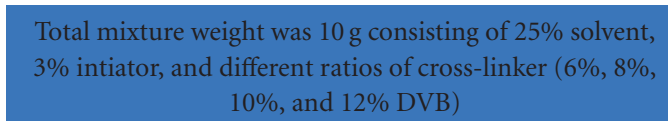

$10 \%$, and $12 \%$ DVB)
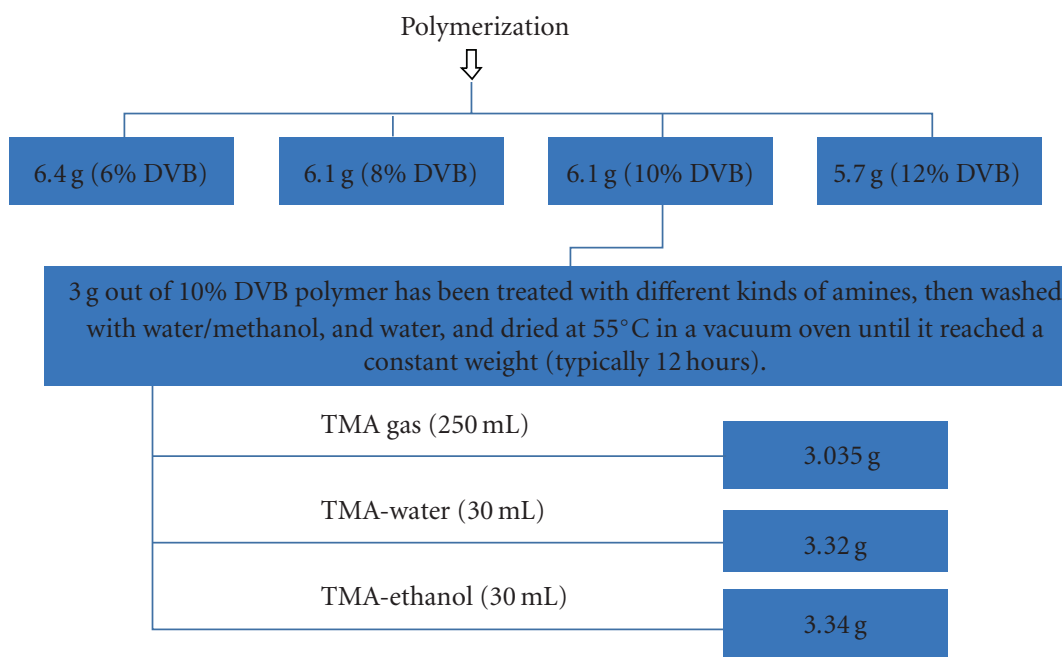

FIGURE 2: Scheme showing the preparing procedures while different factors have been changed. 


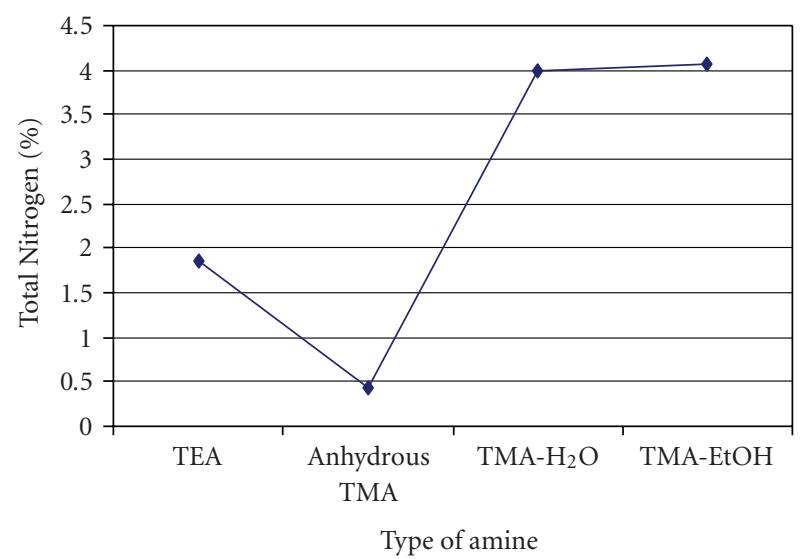

FIGURE 3: Effect of amine type on the nitrogen content (after immersing in $6 \%$ DVB polymer in TMA at room temperature for 4 days).

were washed by successive rinses in deionized water before drying them. The ion exchange capacity (IEC) was calculated as milliequivalents of hydroxide per gram of dry polymer according to the following equation:

$$
\operatorname{IEC}(\mathrm{meq} / \mathrm{g})=\frac{\left[V_{\mathrm{NaOH}} \times\left(N_{1}-N_{2}\right)\right]}{W_{\mathrm{dry}}},
$$

where $V_{\mathrm{NaOH}}$ is the volume of the $\mathrm{NaOH}$ solution $(\mathrm{mL}), N_{1}$ is the initial normality of $\mathrm{NaOH}, \mathrm{N}_{2}$ is its normality after the exchange, and $W_{\text {dry }}$ is the weight of the polymer in the chloride form $(\mathrm{g})$.

2.3.5. Copolymer Testing. Breakthrough experiments were conducted using a VWR Mini-Peristaltic Pump-Variable Flow pump. Dynamic mode tests were conducted using both the novel anion exchanger and commercial beads. About $3 \mathrm{~g}$ of the novel polymer was packed vertically in a 0.4 inch ID column for testing using an initial solution containing $50 \mathrm{ppm}$ of both sulfate and chloride ions with a flow rate of $5 \mathrm{~mL} / \mathrm{min}$. Using a $20 \mathrm{~mL} / \mathrm{min}$ flow rate, solutions containing varying influent concentrations were pumped through both the commercial and novel resins beads. Twenty $\mathrm{ml}$ of effluent was collected repeatedly for analysis. All samples were analyzed by ion chromatography.

\section{Results and Discussion}

3.1. Synthesis of Anion Exchange Copolymers. In this investigation, anion exchange polymers were prepared for potential application in the removal of anions that contribute to the salinity of brackish waters. The synthetic procedure involved copolymerization of vinylbenzyl chloride with divinylbenzene to generate a cross-linked polymer. The chloromethyl groups were subsequently converted to benzyltrimethylammonium groups by reaction with trimethylamine. For comparison purposes, the preparation of the triethylammonium analog was performed using triethylamine as the amination reagent. Several factors in the synthetic procedure were varied to identify a method that maximizes the number

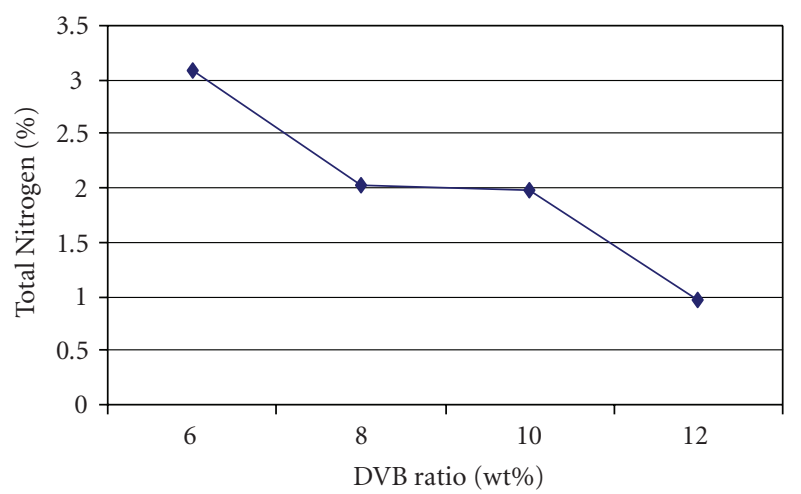

Figure 4: Effect of cross-linker (\%DVB) on the nitrogen content (after immersing in ethanolic TMA at room temperature for 4 days).

of functional groups in the final polymer. These factors included the solvent used for the initial polymer synthesis, the percentage of the cross-linker (divinylbenzene), and the solvent and temperature for the amination reaction.

Figure 3 delineates the degree of functionalization that was achieved in amination of a $11: 1 \mathrm{w}: \mathrm{w}$ VBC:DVB polymer (with $3 \%$ initiator and $25 \%$ solvent). It was found that trimethylamine led to significantly higher amine incorporation than triethylamine, a result that is likely due to steric effects caused by the more bulky ethyl derivative. It was also found that the use of water or ethanol as solvent gave a more complete derivatization reaction. We discovered that trimethylamine/ethanol is best suited for the amination reaction since cracking occurred when water was used as a solvent. Notably, the greatest degree of amination still fell short of the theoretical degree of $6.26 \%$ nitrogen incorporation. This probably results from the polymerization reaction producing benzyl chloride functional groups that are not accessible to the reagent.

The degree of amination can be derived from the nitrogen content of the resin. However, since the presence of variable amounts of water can complicate the comparison of one resin to another. Therefore, combustion analyses were performed for both carbon and nitrogen so that their ratio could be used to remove any influence of variable water content on the analyses. The results, presented in Table 1, show that TMA in ethanol led to the highest incorporation of nitrogen into the resins. Cross-linking reduced the incorporation of nitrogen while elevated temperature increased it. Finally, preparation of the chloromethylstyrene polymer in xylene/tetrahydronaphthalene produced the resin that could be most successfully aminated.

The effect of cross-linking was investigated by preparing polymers with a range of divinylbenzene contents using xylene as a solvent and then aminating with an ethanolic trimethylamine solution. Figure 4 shows that the degree of amination decreased dramatically when the DVB content increased from $6 \mathrm{wt} \%$ up to $12 \mathrm{wt} \%$. The theoretical amount of amine that could be incorporated into the polymer decreases only slightly over this range. The observed decrease is in agreement with the hypothesis that cross-linking produces regions within the polymer that are inaccessible 
TABLE 1: Carbon and nitrogen analysis for the various experimental runs.

(a) Effect of Functionalization agent on the amount of Nitrogen in polymer (after immersing polymer in TEA/EtOH, anhydrous TMA, aqueous TMA and ethanolic TMA at room temp. for 4 days). (VBC $66 \mathrm{wt} \%$, DVB $6 \mathrm{wt} \%$, BPO $3 \mathrm{wt} \%$ and $25 \mathrm{wt} \%$ Solvent (Xylene)

\begin{tabular}{lcc}
\hline Type of amine & TN\% & TC\% \\
\hline TEA/EtOH & 1.86 & 67.5 \\
Anhydrous TMA & 0.42 & 75.1 \\
TMA/ $\mathrm{H}_{2} \mathrm{O}$ & 3.98 & 69.6 \\
TMA/EtOH & 4.06 & 65.4 \\
\hline
\end{tabular}

(b) Effect of cross-linker on the Nitrogen content (after immersing polymer in TMA/EtOH at room temp. for 4 days) (VBC 66, 64, 62, and $60 \mathrm{wt} \%$, DVB 6,8,10,12, respectively, BPO $3 \mathrm{wt} \%$ and $25 \mathrm{wt} \%$ Solvent (Xylene)

\begin{tabular}{lcc}
\hline Divenylbenzene ratio & TN\% & TC\% \\
\hline 6 wt\% DVB & 3.09 & 69.50 \\
8 wt\% DVB & 2.02 & 72.00 \\
$10 w t \%$ DVB & 1.97 & 75.50 \\
12 wt\% DVB & 0.97 & 76.90 \\
\hline
\end{tabular}

(c) Effect of Solvent on the Nitrogen content (after immersing polymer in TMA/EtOH at $60^{\circ} \mathrm{C}$ for 4 days). (VBC (64 wt $\%$, DVB $8 \mathrm{wt} \%$, BPO $3 \mathrm{wt} \%$ and $25 \mathrm{wt} \%$ Solvent)

\begin{tabular}{lcc}
\hline Solvent & TN\% & TC\% \\
\hline THF (Tetrahydrofuran) & 2.12 & 76.10 \\
Xyl. & 4.77 & 66.1 \\
Xyl., THN (1:1) & 5.2 & 67.7 \\
\hline
\end{tabular}

(d) Effect of temperature on the Nitrogen content (after immersing polymer in TMA/EtOH for 4 days). (VBC (64 wt $\%$, DVB $8 \mathrm{wt} \%$, BPO $3 \mathrm{wt} \%$ and $25 \mathrm{wt} \%$ Solvent (THN, Xyl 1:1)

\begin{tabular}{lcc}
\hline Temp & TN\% & TC\% \\
\hline Room temp. & 4.06 & 65.4 \\
$40 \circ \mathrm{C}$ & 4.34 & 61.2 \\
$60 \circ \mathrm{C}$ & 5.2 & 67.7 \\
\hline
\end{tabular}

(e) $\mathrm{TN} \%$ and $\mathrm{TC} \%$ for different DVB ratios at $60^{\circ} \mathrm{C}$ for 4 days in Xyl/THN (1:1) and ethanolic TMA

\begin{tabular}{lcc}
\hline DVB ratio & TN\% & TC\% \\
\hline $6 \%$ & 5.25 & 67.7 \\
$8 \%$ & 5.2 & 67.7 \\
$10 \%$ & 5.05 & 67.5 \\
$12 \%$ & 4.09 & 72.3 \\
\hline
\end{tabular}

to reagents. Such decrease could be due also to the space hindrance of side-methyl, since it may shield some of the amino groups from participating in the ion exchange process [14] or due to the hydrolysis of benzyl chloride groups to benzyl alcohol groups during the polymerization reaction [15].

It is possible that the solvent was used for the initial polymerization reaction to influence the microstructure of the cross-linked co-polymer and thereby affect the amination reaction. Therefore, besides the conventional xylene solvent, tetrahydrofuran (THF) and tetrahydronaphthalene (THN)

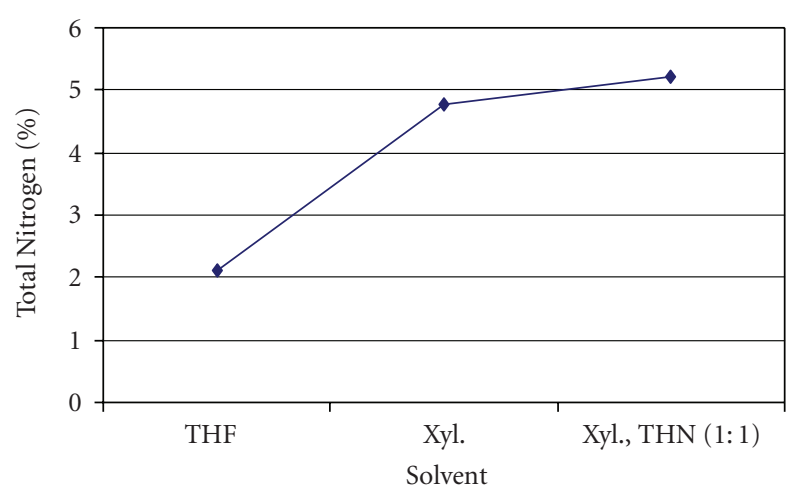

FIGURE 5: Effect of solvent on the nitrogen content after immersing polymer $8 \%$ DVB polymer in amination reagent at $60^{\circ} \mathrm{C}$ for 4 days).

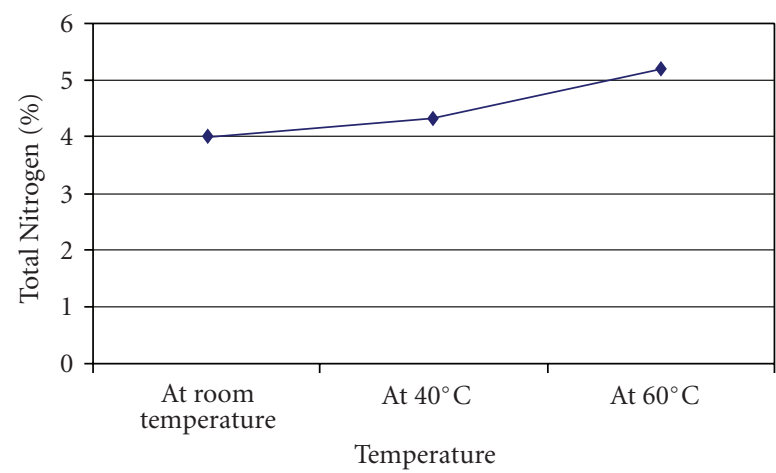

Figure 6: Effect of temperature on the nitrogen content after immersing $8 \%$ DVB polymer in TMA for 4 days).

were employed in this study (Figure 5). THF is a polar solvent that would interact most strongly with the polar chloromethyl groups in the growing polymer, possibly causing them to preferentially locate in solvent-rich pores as the polymer grows. However, this was found not to be the case; the degree of amination of the THF-derived polymer was poorer than the xylene-based product. Assuming that this result may just be predicated on the relative size of the solvent molecules, a much larger solvent, THN, was tested in a $1: 1$ mixture with xylene. It was found that this gave a small increase in the degree of derivatization in the subsequent amination reaction as compared to xylene. Finally, Figure 6 shows that as the temperature of the amination reaction increases the degree of amination increases as well.

3.2. Water Uptake, Nitrogen Content, and Ion Exchange Capacity. The percentage water uptake by an ion exchanger correlates with its hydrophilicity, a parameter that positively influences the rate of ion exchange [14]. It is affected by a variety of factors including the preparation procedure, the degree of cross-linking, and solvent type. To determine the water uptake, the polymer (in chloride form) was soaked in deionized water for $24 \mathrm{~h}$ for swelling and then dried at $50^{\circ} \mathrm{C}$ under vacuum until a stable weight was obtained. The nitrogen content, which has a direct impact on the ion exchange capacity, was determined as a function of 
TABLE 2: Water percent, nitrogen percent, and exchange capacity for polymer prepared with different DVB ratios prepared by polymerization in xylene and THN then aminated using ethanolic trimethylamine.

\begin{tabular}{lcccccc}
\hline DVB \% & $\begin{array}{c}\text { Water } \\
\text { percent } \%\end{array}$ & $\begin{array}{c}\text { Nitrogen } \\
\text { content \% }\end{array}$ & $\begin{array}{c}\text { Exchange } \\
\text { capacity meq/g }\end{array}$ & $\begin{array}{c}\text { wt\% gain after } \\
\text { amination }\end{array}$ & $\begin{array}{c}\text { wt\% loss after } \\
\text { extraction }\end{array}$ & $\begin{array}{c}\text { wt\% loss after treatment } \\
\text { with 0.1 N NaOH }\end{array}$ \\
\hline $6 \%$ & 186 & 5.25 & 2.06 & 27.98 & 27.67 & 3.19 \\
$8 \%$ & 167 & 5.20 & 1.87 & 24.12 & 16.87 & 1.95 \\
$10 \%$ & 146 & 5.05 & 1.78 & 23.41 & 11.73 & 1.30 \\
$12 \%$ & 91 & 4.09 & 0.99 & 16.97 & 9.40 & 1.10 \\
\hline
\end{tabular}

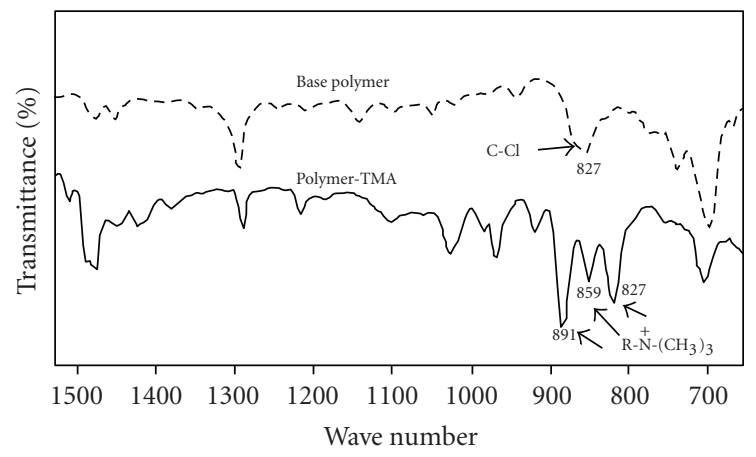

FIGURE 7: FT-IR spectra of the anion exchange polymer.

amination degree. Table 2 shows the variation of water content, nitrogen content, and ion exchange capacity as a function of cross-linkage degrees.

Although the cross-linking degree plays an important role on the degree of amination as well as the ion exchange capacity, the resin containing $10 \%$ DVB was utilized in the subsequent investigation since the yield of polymer was significantly enhanced over polymers prepared with lower amounts of DVB. It was found that there were markedly higher weight losses during the Soxhlet extraction with methanol for the 6 and $8 \%$ polymers, reflecting the generation of higher amounts of soluble, poorly cross-linked polymer in these syntheses. It should be noted also that the observed ion exchange capacities of both $8 \%$ DVB and $10 \%$ DVB are very close to each other. Moreover, the thermal properties of $10 \%$ DVB type are superior to those of the polymers with lower DVB content. Regeneration studies were conducted by taking the samples, with different DVB ratios, soaking them in $0.1 \mathrm{M} \mathrm{NaOH}$ solutions for 48 hours at room temperature, washing them with deionized water, and then vacuum drying and weighing them before they were reused. The regeneration of the resins led to minimal weight losses, especially for the $10 \%$ and $12 \%$ DVB types (Table 2).

3.3. FT-IR Characterization. The FT-IR spectra of VBC$\mathrm{N}\left(\mathrm{CH}_{3}\right)_{3} \mathrm{Cl}$ powders were taken over the range of 400 to $4000 \mathrm{~cm}^{-1}$, but for the analysis of the quaternary ammonium groups, spectra are shown over the range from 800 to $1400 \mathrm{~cm}^{-1}$ in Figure 7. In the base polymer, the absorption bands assigned to the aromatic groups were observed at a range from 1440 to $1695 \mathrm{~cm}^{-1}$. The spectrum has an absorption band at $827 \mathrm{~cm}^{-1}$ that can be attributed to the $\mathrm{C}$ - $\mathrm{Cl}$ stretch of the $\mathrm{CH}_{2} \mathrm{Cl}$ groups that were derived from the vinylbenzyl chloride monomer. For the polymer aminated with trimethylamine, the infrared absorptions due to the quaternary amine were observed at 859 and $891 \mathrm{~cm}^{-1}$. These results show that the amine group was successfully introduced to the base polymer through the functionalization process. These results are in agreement with the literature [16]. There is also a residual peak due to $\mathrm{C}-\mathrm{Cl}$ stretch of the unreacted benzylchloride groups.

3.4. Thermal Properties. The thermal stabilities of the base polymer, $\mathrm{P}-\mathrm{N}\left(\mathrm{CH}_{3}\right)_{3} \mathrm{Cl}, \mathrm{P}-\mathrm{N}\left(\mathrm{CH}_{3}\right)_{3} \mathrm{OH}$, and DOWEX MONOSPHERE 550A $(\mathrm{OH})$ resins were studied under air atmosphere by thermal gravimetric analysis. The results, presented in Figure 8, show that the samples possess three main weight loss peaks. As mentioned in previous investigations $[17,18]$, the first weight-loss step at low temperature is mainly due to evaporation of residual ethanol, water, and/or other solvents. The second weight-loss step is the decomposition of pendant functional groups such as quaternary ammonium or chloromethyl groups. The third degradation is ascribed to the polymer backbone decomposition/oxidation. The base polymer exhibited gradual weight loss until approximately $200^{\circ} \mathrm{C}$ when more rapid decomposition began. The aminated polymer in the chloride form showed an increase in thermal stability, showing an onset of thermal decomposition at $208^{\circ} \mathrm{C}$. When the thermal stability of the $\mathrm{OH}$ form of the prepared polymer was compared to that of the commercial one (DOWEX 550A) it was found that the polymer degradation onset occurred at a significantly elevated temperature $\left(165^{\circ} \mathrm{C}\right)$ that it did for the DOWEX resin $\left(149^{\circ} \mathrm{C}\right)$.

3.5. Breakthrough Curve. The new anion exchange resin was tested in its hydroxide form in a breakthrough experiment when it was challenged with a solution containing $50 \mathrm{ppm}$ of both sulfate and chloride ions (Figure 9). The experiment was performed at a constant $5 \mathrm{~mL} / \mathrm{min}$ flow rate using $3 \mathrm{~g}$ copolymer in a 0.4 inch (ID) column and a bead depth of $5.75 \mathrm{~cm}$ dry and $7.5 \mathrm{~cm}$ wet.

The polymer is more efficient at removing sulfate than chloride because of the selectivity preference for sulfate over chloride. As a result, sulfate replaces both hydroxide and chloride, while chloride replaces only hydroxide sites at these concentrations. Functionally, this is due to the strongly basic functional groups (benzyltrimethylammonium) which are highly selective toward divalent ions [9]. Once hydroxide 


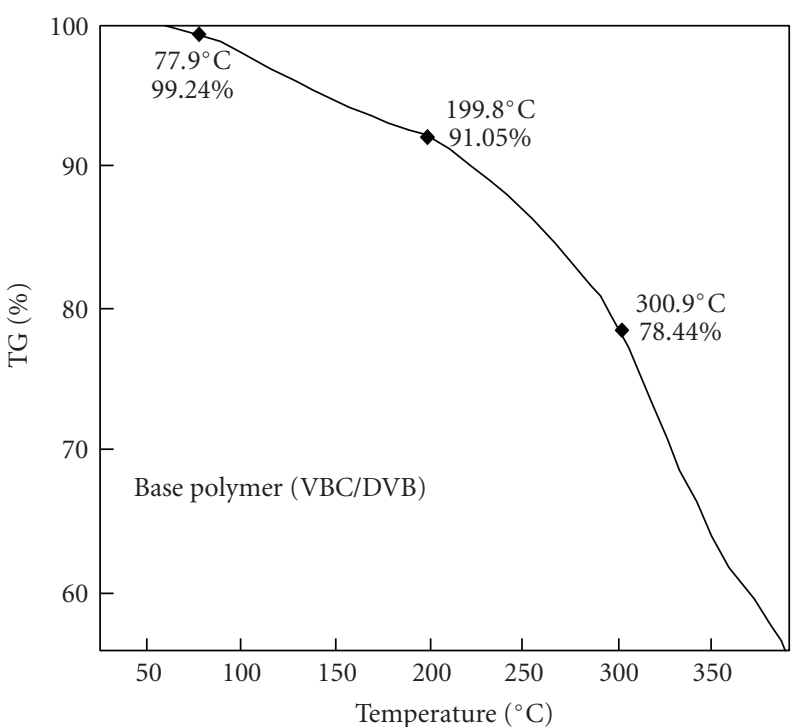

(a)

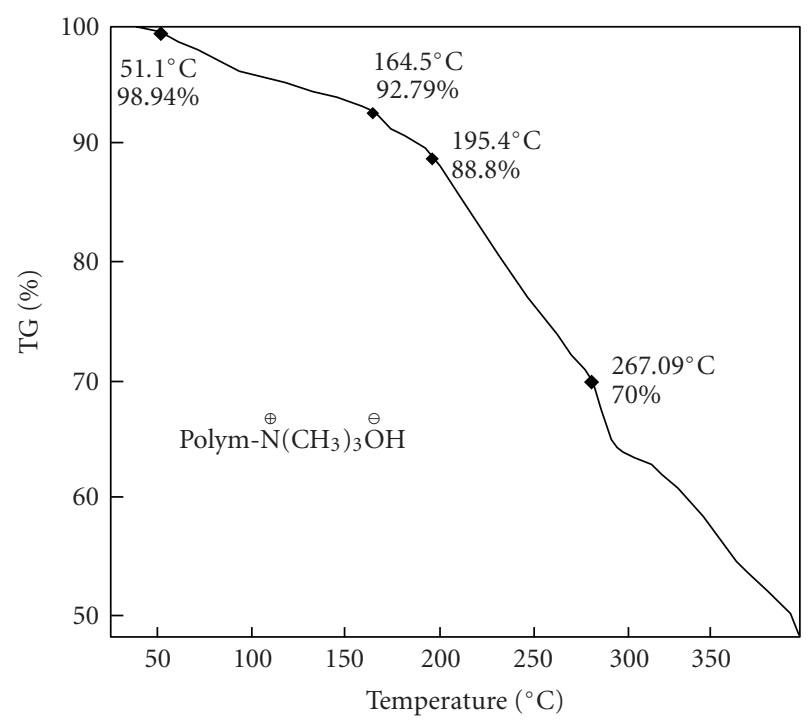

(c)

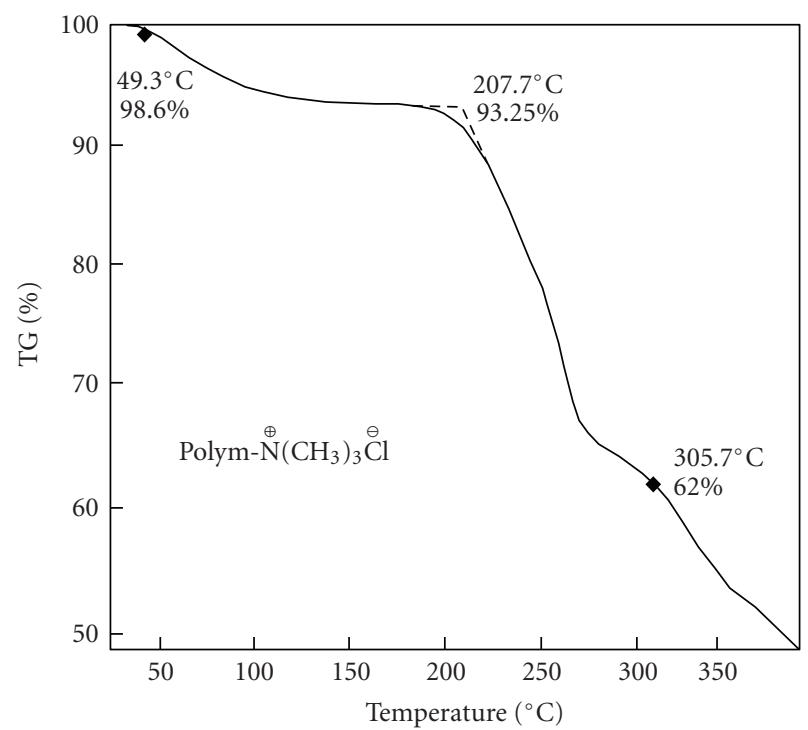

(b)

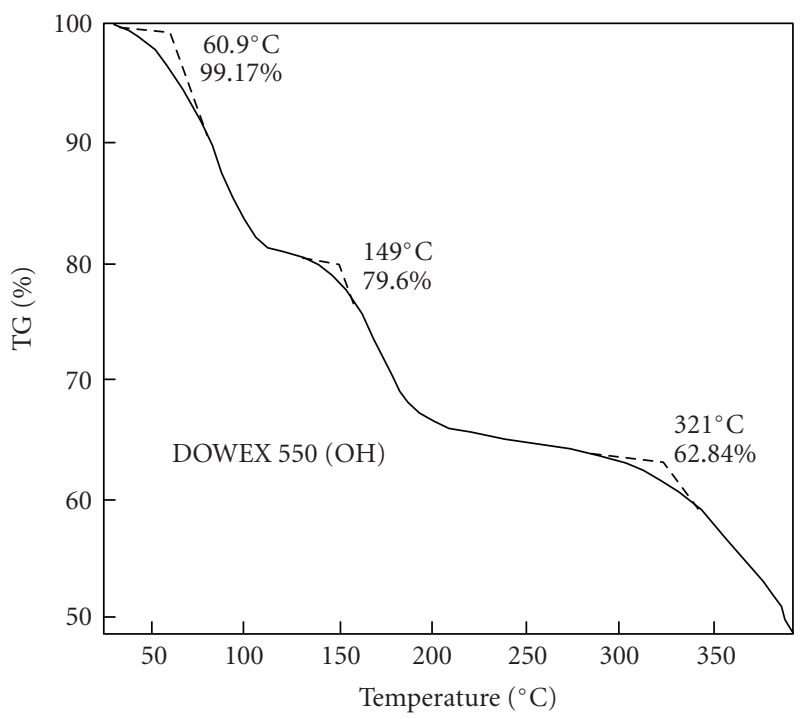

(d)

Figure 8: Thermogravimetric curves $\left(5^{\circ} \mathrm{C} / \mathrm{min}\right.$, air) for base polymer, $\mathrm{P}-\mathrm{N}\left(\mathrm{CH}_{3}\right)_{3} \mathrm{Cl}, \mathrm{P}-\mathrm{N}\left(\mathrm{CH}_{3}\right)_{3} \mathrm{OH}$, and DOWEX 550A $(\mathrm{OH})$.

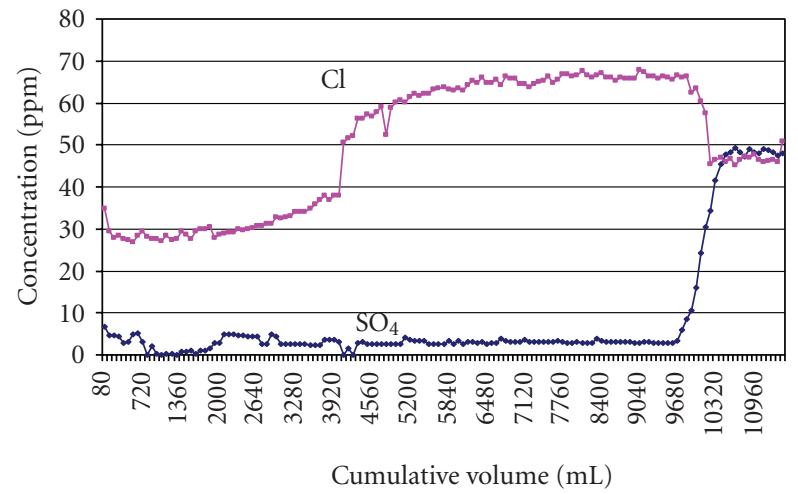

FIGURE 9: Breakthrough results using $50 \mathrm{ppm} \mathrm{SO}_{4}$ and $\mathrm{Cl}$. sites are exhausted, chloride ions freely pass through the membrane, and the effluent concentration of chloride starts to rise. This rise continues past the chloride feed concentration because the effluent includes chloride in the feed plus chloride displaced by sulfate. Eventually, when the polymer fills with sulfate, the chloride concentration returns to its initial value.

The performance of the novel anion exchange resin was further tested with a solution containing $50 \mathrm{ppm}$ of each of several anions $\left(\mathrm{Cl}^{-1}, \mathrm{SO}_{4}{ }^{-2}, \mathrm{NO}_{3}{ }^{-1}\right.$, and $\left.\mathrm{PO}_{4}{ }^{-3}\right)$, and the results were compared to those for DOWEX 550A resin (Figures 10 and 11). It was shown that the commercial polymer definitely exceeded the new one in the efficiency in removing chloride ions. However, the newly synthesized 


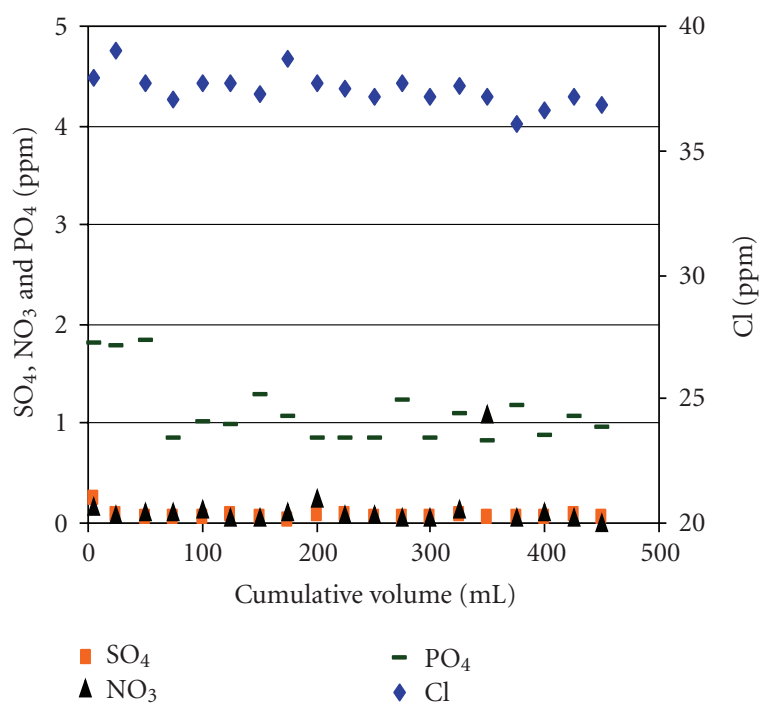

FIGURE 10: Breakthrough results for the novel ion exchanger using $50 \mathrm{ppm} \mathrm{Cl}, \mathrm{SO}_{4}, \mathrm{NO}_{3}$, and $\mathrm{PO}_{4}$.

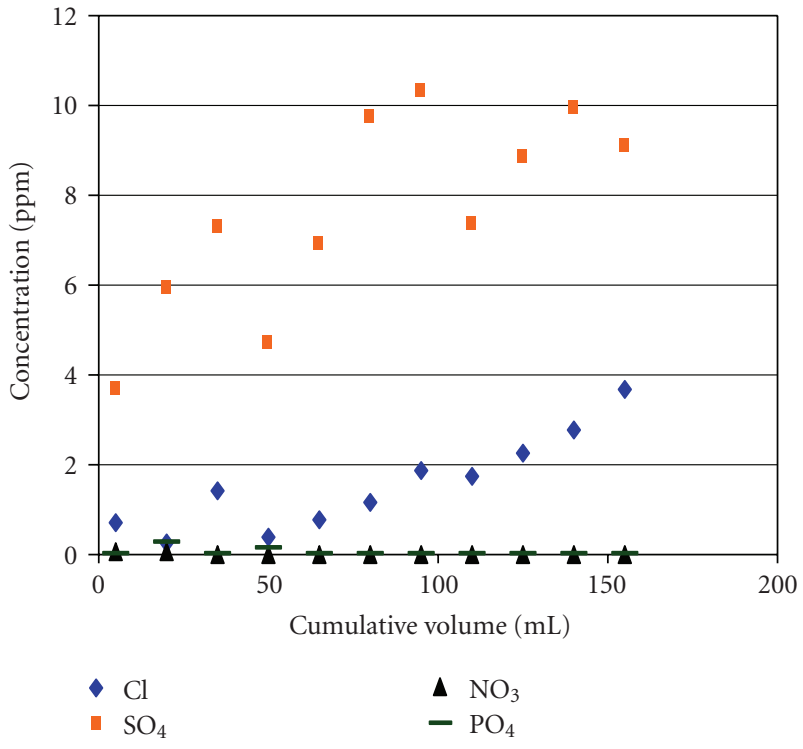

FIGURE 11: Breakthrough results for DOWEX ion exchanger using 50 ppm Cl, $\mathrm{SO}_{4}, \mathrm{NO}_{3}$, and $\mathrm{PO}_{4}$.

anion exchange resin performed well with all of the other anions tested and was clearly superior to the commercial products in removing sulfate from water. Figure 12 shows that even in the presence of high chloride concentrations, the new ion exchange resin performs well in removing sulfate.

In Figure 13, the results from treatment of a naturallyoccurring groundwater sample with the novel anion exchange resin are presented. The electrical conductivity of the solution decreased from $4670 \mu \mathrm{s} / \mathrm{cm}$ to about $2900 \mu \mathrm{s} / \mathrm{cm}$. This change is likely due to a reduction of $\mathrm{pH}$ as the other anions are replaced with hydroxide ions. The concentrations of chloride and sulfate present in the water sample were decreased significantly to $600 \mathrm{ppm}$ and $3 \mathrm{ppm}$ from initial

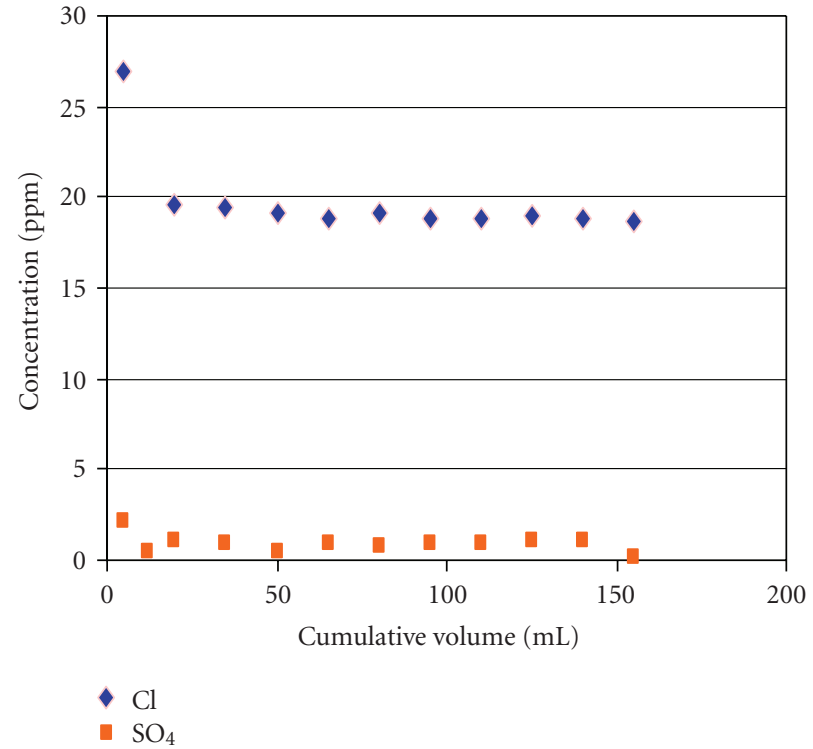

FIGURE 12: Breakthrough results for prepared ion exchanger using $50 \mathrm{ppm} \mathrm{Cl}$ and $10 \mathrm{ppm} \mathrm{SO}_{4}$.

concentrations of chloride and sulfate of $1500 \mathrm{ppm}$ and 300 ppm, respectively.

\section{Conclusion}

Several methods were used to prepare anion exchange polymers with higher cross-linking and reasonable ion exchange capacity. The type of solvent influenced the degree of amination when the cross-linker ratio was increased. The anion exchanger prepared in this investigation had improved thermal stability as compared to commercial resins. The results from the ion-exchange experiments suggest that this kind of anion exchanger is more selective for divalent anions 


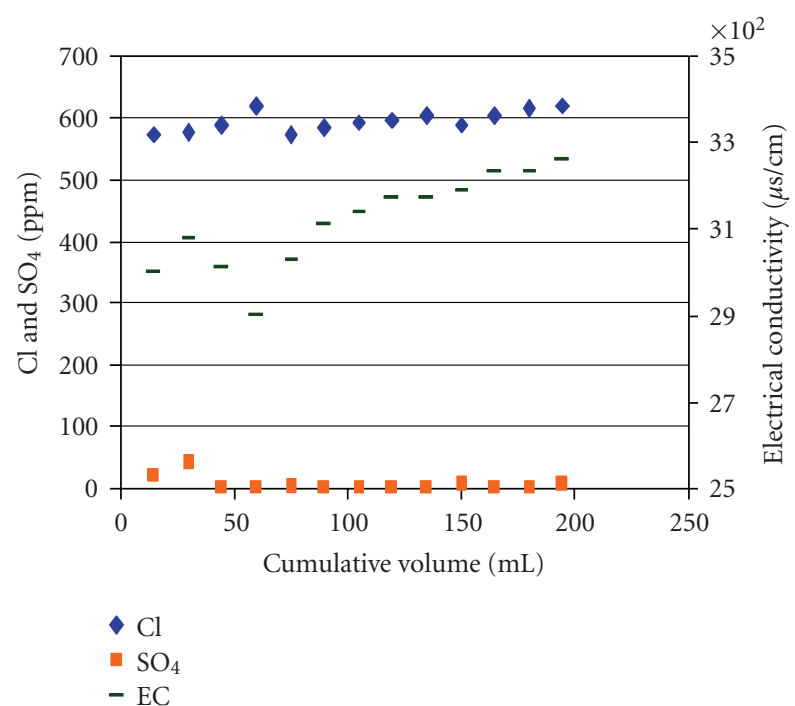

FIGURE 13: Breakthrough results for prepared ion exchanger using groundwater sample.

rather than monovalent anions especially chloride. However, it works well with chloride when the concentrations of other divalent anions (e.g., sulfate) were significantly lower than that of the chloride.

\section{Acknowledgment}

The authors wish to thank Dr. Eliot Atekwana, Associate Professor, Boone Pickens School of Geology, Oklahoma State University for allowing them use lab to run many samples on the ion chromatograph, and they really value his cooperation.

\section{References}

[1] T. Sata, "Ion exchange membranes and separation processes with chemical reactions," Journal of Applied Electrochemistry, vol. 21, no. 4, pp. 283-294, 1991.

[2] X. F. Zeng and E. Ruckestain, "Novel method of preparing microporous membrane by selective dissolution of chitosan/polyethylene glycol blend membrane," Biotechnology Progress, vol. 15, 1003 pages, 1999.

[3] C. M. Wu, T. W. Xu, and W. H. Yang, "Fundamental studies of a new hybrid (inorganic-organic) positively charged membrane: membrane preparation and characterizations," Journal of Membrane Science, vol. 216, no. 1-2, pp. 269-278, 2003.

[4] K. W. Pepper, H. M. Paisley, and M. A. Young, "Properties of ion-exchange resins in relation to their structure. Part VI. Anion-exchange resins derived from styrene-divinylbenzene copolymers," Journal of Applied Chemistry, pp. 4097-4105, 1953.

[5] S. Laskin, R. T. Drew, and V. Cappiello, "Inhalation carcinogenicity of alpha halo ethers," Archives of Environmental Health, vol. 30, no. 2, pp. 70-72, 1975.

[6] The Carcinogenic Substances Regulations, Statutory Instrument, no. 879, HMSO, London, UK, 1967.

[7] R. B. Wuchter, Direct Acylaminomethylation of Aromatic Polymers, vol. 37, US Patent, Alexandria, VA, USA, 1974.
[8] K. Dorfner, Ion Exchangers, Walter de Gruyter, Berlin, Germany, 1991.

[9] L. Dominguez, J. Economy, K. Benak, and C. L. Mangun, "Anion exchange fibers for arsenate removal derived from a vinylbenzyl chloride precursor," Polymers for Advanced Technologies, vol. 14, no. 9, pp. 632-637, 2003.

[10] J. D. Giffin, "Chloromethylation of polystyrene," Industrial \& Engineering Chemistry, vol. 44, no. 11, pp. 2686-2693, 1952.

[11] E. O. Kartinen Jr. and C. J. Martin, "An overview of arsenic removal processes," Desalination, vol. 103, no. 1-2, pp. 79-88, 1995.

[12] L.-L. Horng and D. Clifford, "The behavior of polyprotic anions in ion-exchange resins," Reactive \& Functional Polymers, vol. 35, no. 1-2, pp. 41-54, 1997.

[13] K. Vaaramaa and J. Lehto, "Removal of metals and anions from drinking water by ion exchange," Desalination, vol. 155, no. 2, pp. 157-170, 2003.

[14] H. $\mathrm{Xu}$ and $\mathrm{X} . \mathrm{Hu}$, "Preparation of anion exchangers by reductive amination of acetylated crosslinked polystyrene," Reactive \& Functional Polymers, vol. 42, no. 3, pp. 235-242, 1999.

[15] S. Subramonian, "Anion-exchange resins from vinylbenzyl choride: control of hydrolysis during polymerization," Reactive \& Functional Polymers, vol. 29, no. 2, pp. 129-133, 1996.

[16] Y.-J. Choi, M.-S. Kang, J. Cho, and S.-H. Moon, "Preparation and characterization of LDPE/polyvinylbenzyl trimethyl ammonium salts anion-exchange membrane," Journal of Membrane Science, vol. 221, no. 1-2, pp. 219-231, 2003.

[17] S. M. Kolhe and A. Kumar, "Preparation of strong base anion exchange membrane using ${ }^{60} \mathrm{Co}$ gamma radiation," Radiation Physics and Chemistry, vol. 74, no. 5, pp. 384-390, 2005.

[18] J. Fang and P. K. Shen, "Quaternized poly(phthalazinon ether sulfone ketone) membrane for anion exchange membrane fuel cells," Journal of Membrane Science, vol. 285, no. 1-2, pp. 317322, 2006. 

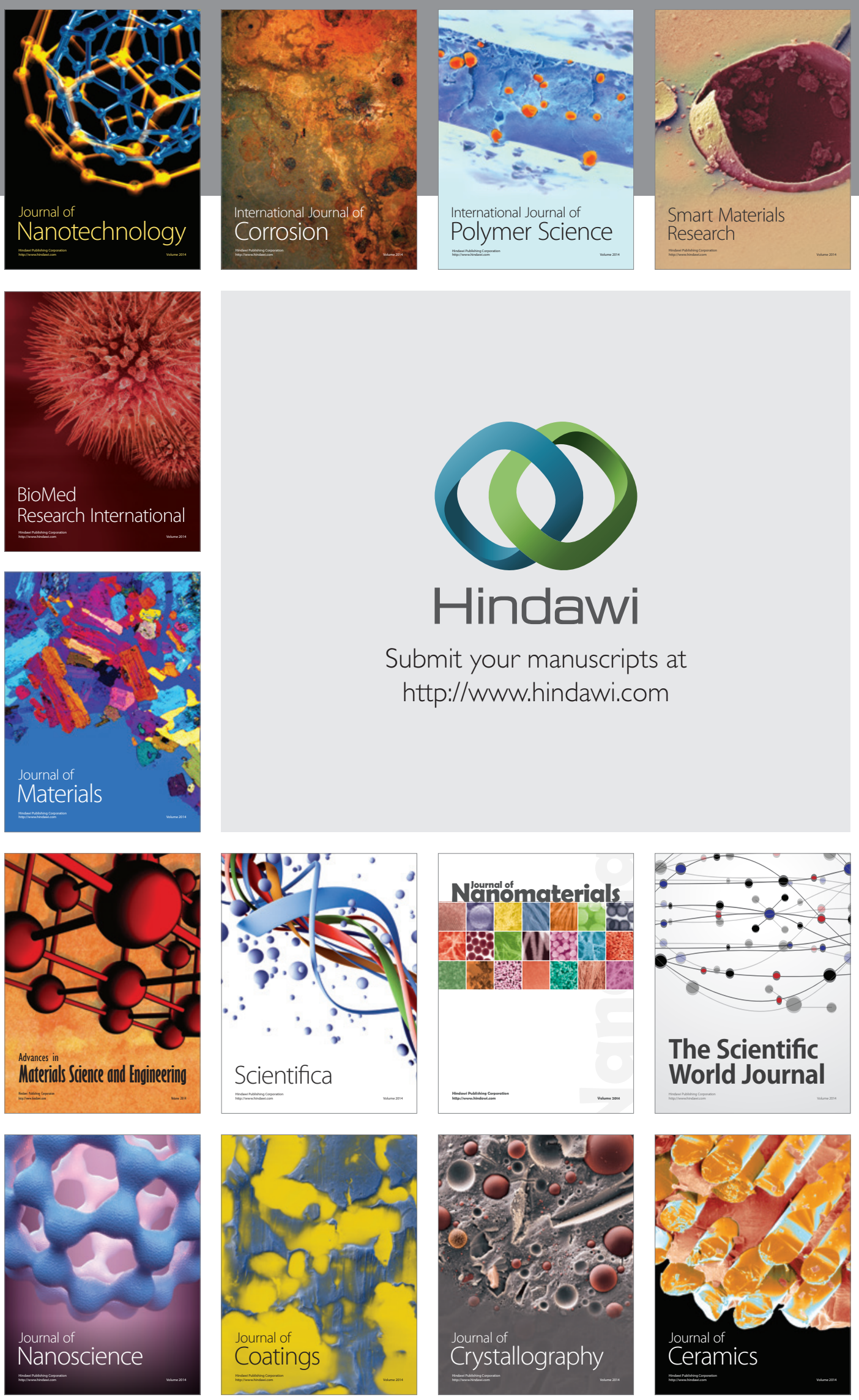

The Scientific World Journal

Submit your manuscripts at

http://www.hindawi.com

\section{World Journal}

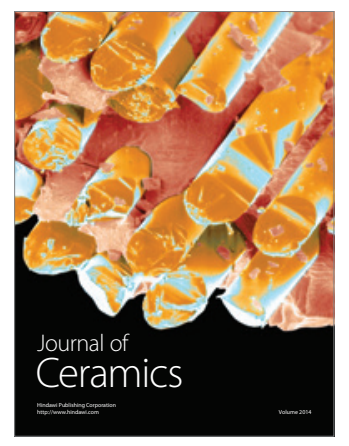

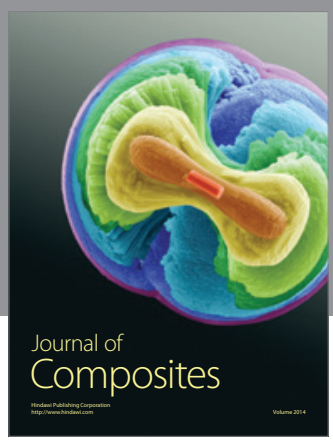
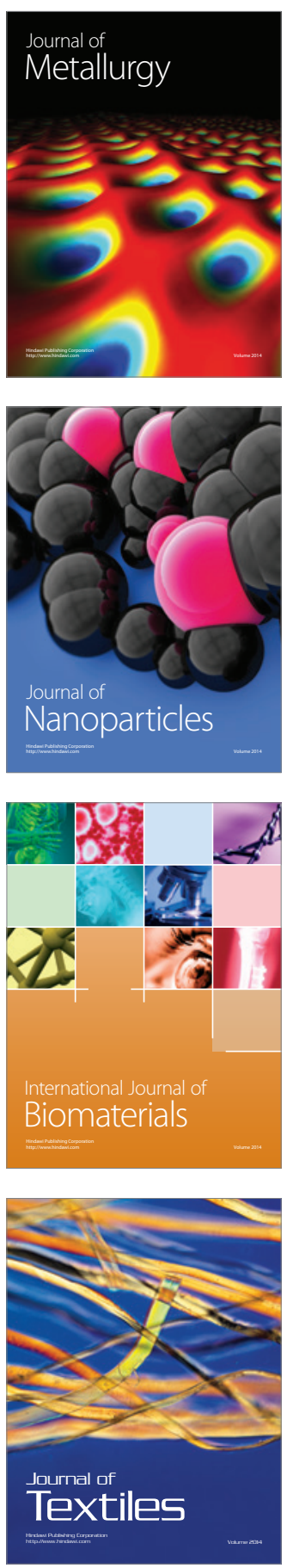